\title{
Comparison of Resting-State Brain Activation between Healthy Normal and Low Auditory-Verbal Working Memory Capacity Participants
}

\section{Nur Ruzainah Gafoora, Ahmad Nazlim Yusoff ${ }^{\mathrm{b}, *}$, Elza Azri Othman ${ }^{\mathrm{c}}$, Nurul Hanim Nasaruddin ${ }^{d}$}

aDiagnostic Imaging and Radiotherapy Program, Faculty of Health Science, Universiti Kebangsaan Malaysia, Jalan Raja Muda Abdul Aziz, 50300 Kuala Lumpur Malaysia; b Center for Diagnostic, Therapeutic and Investigative Studies, Faculty of Health Science, Universiti Kebangsaan Malaysia, Jalan Raja Muda Abdul Aziz, 50300 Kuala Lumpur, Malaysia; ' School of Medical Imaging, Faculty of Health Sciences, Universiti Sultan Zainal Abidin, Gong Badak Campus, 21300 Kuala Nerus, Terengganu, Malaysia; ${ }^{d}$ Department of Cognitive Science, Faculty of Cognitive Science and Human Development, Universiti Malaysia Sarawak, 94300 Kota Samarahan, Sarawak, Malaysia.

Abstract Working memory (WM) capacity is the ability to maintain attention and store information briefly in the mind. However, each individual has a limited WM capacity that varies from one person to another. An individual can be categorized as having either normal or low WM capacity. This study aimed to evaluate and compare brain activations of healthy individuals with low and normal auditory-verbal WM capacity. A total of 39 healthy male young adults were recruited from local universities for this study. They were categorized into the normal and low auditory-verbal WM capacity group based on their score in the Malay Version of Auditory Verbal Learning Test (MVAVLT). All participants underwent resting-state functional magnetic resonance imaging (rs-fMRI) scans. The functional data were analyzed using Statistical Parametric Mapping (SPM) and Wake Forest University (WFU) Pickatlas softwares. Brain activations and resting-state amplitude fluctuation (RsAF) were contrasted between groups to determine whether there were any significant differences caused by the different auditory-verbal WM capacity. The findings indicated that the low auditory-verbal WM capacity group showed significantly higher cortical activations in the left lingual gyrus, bilateral middle temporal gyrus, left calcarine, left superior

*For correspondence: nazlimtrw@ukm.edu.my Received: 11 Mac 2021 Accepted: 20 Dec 2021

(C) Copyright Gafoor et al. This article is distributed under the terms of the Creative Commons

Attribution License, which permits unrestricted use and redistribution provided that the original author and source are credited frontal gyrus, and left precuneus as compared to normal auditory-verbal WM capacity group. It is suggested that the higher activation of these brain areas in low verbal-auditory WM capacity participants was attributed to the lower neural adaptability of the brain at rest.

Keywords: Auditory, fMRI, healthy adults, resting-state, verbal-auditory working memory capacity.

\section{Introduction}

Working memory (WM) is defined as the cognitive ability to temporarily maintain and manipulate information [1]. The WM is crucial when performing tasks involving higher cognitive functions. The central executive is the key component of WM and controls one's attention level [2]. Under the central executive, 
there are two components that temporarily maintain and manipulate the information received by the brain; the visuospatial sketchpad and phonological loop. These temporary storages are independent of one another and are separated based on the type of processed information. While the visuospatial sketchpad component processes visual information, the phonological loop component processes auditory information [2]. This study focuses on the phonological loop component because it forms the basis of auditory-verbal WM and involved in the maintenance of auditory information.

The phonological loop is further divided into two subcomponents; the phonological store and articulatory rehearsal [2]. The phonological store is time-limited storage that stores speech-based information for two seconds before the information decay. On the other hand, the articulatory rehearsal refreshes the auditory information by recirculating them into the phonological store before they decayed. This process is done by constantly reciting the words in the mind (inner speech). A previous study has associated the phonological loop with the Broca's and Wernicke's areas [3]. Broca's area is located at posterior inferior frontal gyrus (Broadman areas 44 and 45) with left hemisphere dominance [3]. Broca's area is mainly involved in language production and comprehension [4]. Although the exact function of the Broca's area in speech production remains unclear, it has been suggested that this area is involved in motor function [5]. The Broca's area has also been associated with verbal WM, grammar, syntax, fluidity, language repetition, gesture, and interpreting other's action [6]. On the other hand, the Wernicke's area is located on the left posterior Sylvian fissure synonymous to left posterior superior temporal gyrus (pSTG) and posterior supramarginal gyrus [3]. These areas are commonly known to be involved in speech comprehension [3].

Apart from briefly storing the information in the mind, the WM also manipulates the stored information. Manipulation of information within WM helps an individual to prepare an organized step of actions before executing them. The dorsolateral prefrontal cortex (DLPFC) is the key brain area of the WM that manipulates information. The DLPFC is interlinked with the brain's anterior and posterior cortical and subcortical area [7]. This widespread cerebral network allows DLPFC to maintain and manipulate the stored information effectively. Disruption of DLPFC's activity and coordination has been associated with schizophrenia [8], early-stage Alzheimer's disease [9], and Attention Deficits Hyperactivity Disorder (ADHD) [10]. All these psychiatric and neurological disorders are related to low WM capacity [11, 12].

It is worthy to note that the WM has limited capacity, whereby the information cannot be stored and manipulated endlessly in the mind. The WM may retain a maximum of four to seven items at a given time [13]. However, each individual has different WM capacities, which explained individual differences in general fluid intelligence [14], attentional control [15], mathematical or arithmetic ability [16], comprehension [17], learning [18], and reading [19]. Similar age group also showed variations of WM capacity, indicating that the WM capacity was not affected only by the maturation of frontal cortices [20]. WM deficits reduce WM domains' ability and quality of life [20, 21]. Hence, identifying the neural mechanisms of WM plays a vital role to target certain brain areas to drive the neural processes back to a more optimal state and improve the behavior along with it.

In this study, resting-state brain activations of normal and low auditory-verbal WM capacity participants were measured and compared. This study aimed to determine whether there were any differences in resting-state brain activations that cause individuals to have reduced ability to encode, maintain, and retrieve auditory information. Normal auditory-verbal WM capacity participants have been associated to the efficiency of neural usage, that is ability to adapt the neural activation (increase or decrease) according to task requirement [22]. As in a resting condition, cognitive demands are low and no task is involved. For that, it was hypothesized that normal auditory-verbal WM participants to have lower restingstate brain activations than low auditory-verbal WM capacity participants. Lower resting-state brain activations indicate better brain organisation in normal auditory-verbal WM capacity participants. Structure and function of each activated brain areas were discussed. 


\section{Materials and methods}

\section{Participants}

The data were obtained from a previous study [23] approved by the Institutional Ethics Committee of the Universiti Kebangsaan Malaysia (UKM/PPI/111/8/JEP-2017-117) and Malaysia Medical Research and Ethics Committee (NMRR-17-56-33800). Thirty-nine healthy male young adults were recruited from local universities in Malaysia for this study. Participants were briefed about the study's aims and procedures before obtaining their written informed consent. All participants were native Malay speakers, right-handed and had no history of psychotic or neurological disorders. The participants were also free from the use of psychoactive medications and had no MRI contraindications (e.g., metallic implants, surgical clips and aneurysm clips).

\section{WM Capacity Measures}

All participants performed the Malay Version of Auditory Verbal Learning Test (MVAVLT) and were grouped into the normal and low auditory-verbal WM capacity groups based on their scores. Every participant was given clear instruction in Malay as adapted from a previous study [24]. The participants were asked to listen carefully, memorize, and recall as many words as possible from a 15 words list being read aloud at an approximately one-second interval by the experimenter. The procedure was repeated for five trials (trial $\mathrm{A} 1$ to $\mathrm{A} 5$ ) and there was no time limit for the participants to respond. In each trial, the assessment ended when the participants were unable to recall any more words. The final score was calculated by summing each score from each trial (one mark for each correct word). Hence, a maximum score was 75 (each trial $=15$ scores). Participants who managed to score within the upper half of the maximum score (scores of 38 to 75) were categorized into normal auditory-verbal WM capacity group, whereas participants scored within the lower half of the range (scores 1-37) were categorized into low auditory-verbal WM capacity group [25].

\section{MRI Acquisition}

Participants were instructed to keep their heads still and remained calm during the MRI scans. They were told to remain awake (not sleeping) and focused on a fixation point ('X' symbol) inside the MRI gantry throughout the scanning session. They were also told not to think or mind-wandering because the brain network might be different $[26,27]$. The resting-state functional magnetic resonance imaging (rsfMRI) scans were performed using clinically approved 3T Siemens Magnetom Verio at Universiti Kebangsaan Malaysia Medical Centre (UKMMC) [26]. The first few scans were automatically removed for each participant to ensure signal stabilisation by eliminating the magnetic saturation effect [28] and allowed the participants to familiarize with the MRI noise [29]. Functional T2*-weighted images were acquired for nine minutes and 33 seconds using gradient-echo echo-planar imaging (GRE_EPI) or blood oxygenation level-dependent (BOLD) imaging technique. The imaging parameters included echo time $(T E)=29 \mathrm{~ms}$, repetition time $(T R)=2 \mathrm{~s}$, flip angle $(\alpha)=75^{\circ}$, slice thickness $=3.5 \mathrm{~mm}$, slice gap $=1.05$ $\mathrm{mm}$, field of view $(\mathrm{FOV})=240 \mathrm{~mm}$, matrix size $=64 \times 64$, voxel size $=3.75 \times 3.75 \times 4.55 \mathrm{~mm}$ and the number of scans $=200$.

\section{Image Pre-processing}

A total of 150 functional scans (motion corrected) were analyzed using Statistical Parametric Mapping (SPM12). Firstly, the images were checked randomly for any magnetic field distortion artifacts. Slice timing correction was done using the first slice as a reference. Next, head motion realignment was done to minimize the effects of participants' movement on overall signal intensity because signal variation from movement may interfere the hemodynamic response. There were six realignment parameters, including the translation motion of the head $(x, y, z)$ and rotational motion of the head (pitch, roll and yaw). After realigning the image data, spatial normalisation of the images was done to match the EPI template provided by the Montreal Neurological Institute (MNI). The images were smoothed using an $8 \mathrm{~mm}$ fullwidth-at-half-maximum (FWHM) Gaussian kernel to increase the signal-to-noise ratio. 


\section{Brain Activations Measures Using a General Linear Model (GLM)}

The human brain at rest naturally exhibits low-frequency fluctuations (LFF), making it possible to compute a general linear model (GLM). To incorporate the LFF into GLM, the function must be approximated using a summation of mathematical components of sine and cosine functions with different frequencies [30]. The LFF range for this study was from 0.008 to $0.1 \mathrm{~Hz}$. The standard cut-off brain frequency at rest is $0.008 \mathrm{~Hz}$, removing any other low-frequency fluctuations caused by breathing, cardiac effects, and aliased biorhythms. Previous studies believed that the LFF of resting-state BOLD fMRI signal is linked to the brain's neuronal activity [31]. A previous study proposed that resting-state brain activity may correspond to how a person responds to external stimuli and perform a task [32]. To model the LFF, a combination of mathematical functions such as sine and cosine functions of different frequencies that show a resemblance of LFF, can be used. The functions, also known as Fourier basis set [30], can be incorporated into the general linear model (GLM) as shown by the design matrix in Figure 1 (a). This Fourier basis set with $\mathrm{Ns}=47$ is thought to be suitable to model brain responses during restingstate which is assumed to oscillate between $0.008-0.1 \mathrm{~Hz}$ [30]. In Figure 1(a), horizontal columns 1 to 94, denoted as parameters, represent the Fourier basis set with $90^{\circ}$ phase delay oscillating at $0.008 \mathrm{~Hz}$ to $0.1 \mathrm{~Hz}$ in step of $0.002 \mathrm{~Hz}$. Column 95 to column 100 represent the movement related parameters. The last column, column 101 represents the effects that may be caused by other factors. On the other hand, vertical lines in the design matrix, denoted as images, are number of scans. In this study, the 150 rs-fMRI functional scans for each of the participants were analyzed to produce a single-subject design matrix. The GLM shown in Figure 1(a) was then estimated and the sum of squared weighted beta images (the ESS images) for individual participant was obtained using the F statistics. The ESS images obtained from individual-subject analysis were entered into differential group analysis conducted using an independent sample t-test to compare between the low and normal auditory-verbal WM capacity participants. The design matrix used is shown in Figure 1(b). The area, sub-area, the anatomical functions, voxel value, and peak coordinates of the activations for comparisons of Low > Normal and Normal > Low thresholded at corrected and uncorrected $p$ values are recorded and tabulated. The confirmation of the areas of activation, if any, was obtained from an ROI analysis using a MATLAB-based WFU Pick Atlas toolbox (Wake Forest University, North Carolina, USA).

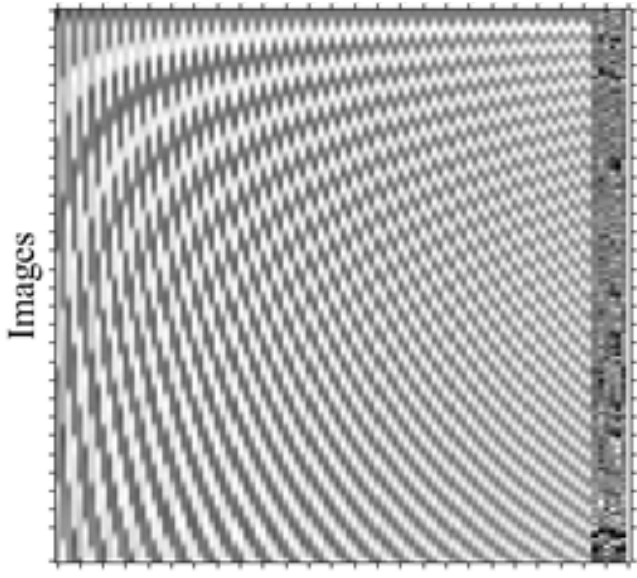

Parameters

(a)

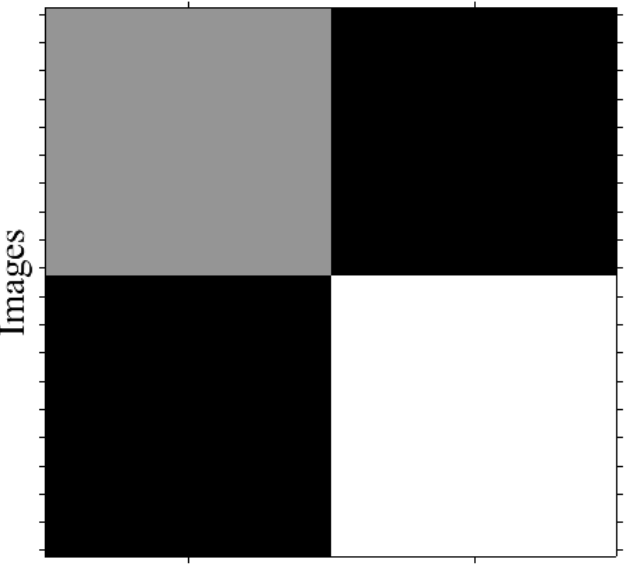

Parameters

(b)

Figure 1. Design matrix used for a) individual subject and b) group differential analyses

\section{Resting-State Amplitude Fluctuations (RsAF)}

Signal change for resting-state fMRI data is best studied using the amplitude of signal fluctuation or Resting-State Amplitude Fluctuation (RsAF). The coordinates $(x, y, z)$ of the voxel with the highest signal intensity from group differential activation map generated by SPM were used to extract this signal 
fluctuation from individual participant. The coordinates are shown in Table 1. Signals were extracted from a spherical node with a radius of $8 \mathrm{~mm}$ from the seven DMN regions shown in Table 1 with their maximum intensity coordinates as the center of the sphere. The fluctuation amplitudes for every region (Table 1) from every participant were then averaged and recorded. An example of an RsAF obtained from an activated region is shown in Figure 2. The Statistical Packages for Social Sciences (SPSS) was used to compare the RsAF of every region between Low and Normal auditory-verbal WM groups. Comparisons were made at $95 \% \mathrm{Cl}$ and the results were considered significant if the $p$ value obtained is smaller than 0.05 .

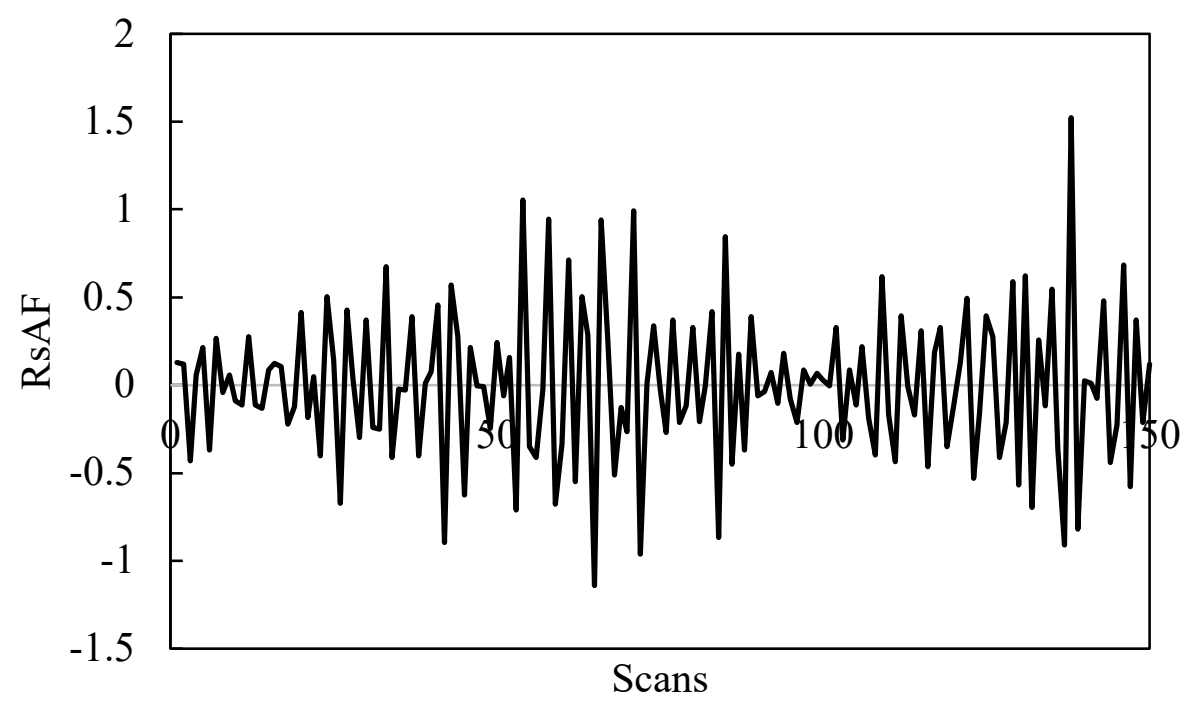

Figure 2. An example of a resting-state amplitude fluctuation over 150 scans

\section{Results}

\section{Working Memory (WM) Capacity Measures}

From the MVAVLT, 20 participants scored above 38 and they were grouped in the normal auditory-verbal WM capacity. The remaining 19 participants scored below 38 and were grouped as low auditory-verbal WM capacity.

\section{Brain Activations}

Based on the group differential analysis using a Normal > Low contrast, no voxel survived the corrected $(p=0.05)$ and uncorrected $(p=0.001)$ thresholds. For Low $>$ Normal contrast, no voxel survived the corrected threshold $(p=0.05)$. However, as shown in Figure 3, several brain areas showed significant differences at the uncorrected threshold $(p<0.001)$. For a better visualisation and to exclude trivial effects, the activation was spatially thresholded at $k=100$ voxels. These areas were the left lingual gyrus, left thalamus, bilateral middle temporal gyrus (MTG), left calcarine, left superior frontal gyrus (SFG), and left precuneus. Further details about these activated brain areas are summarized in Table 1. 

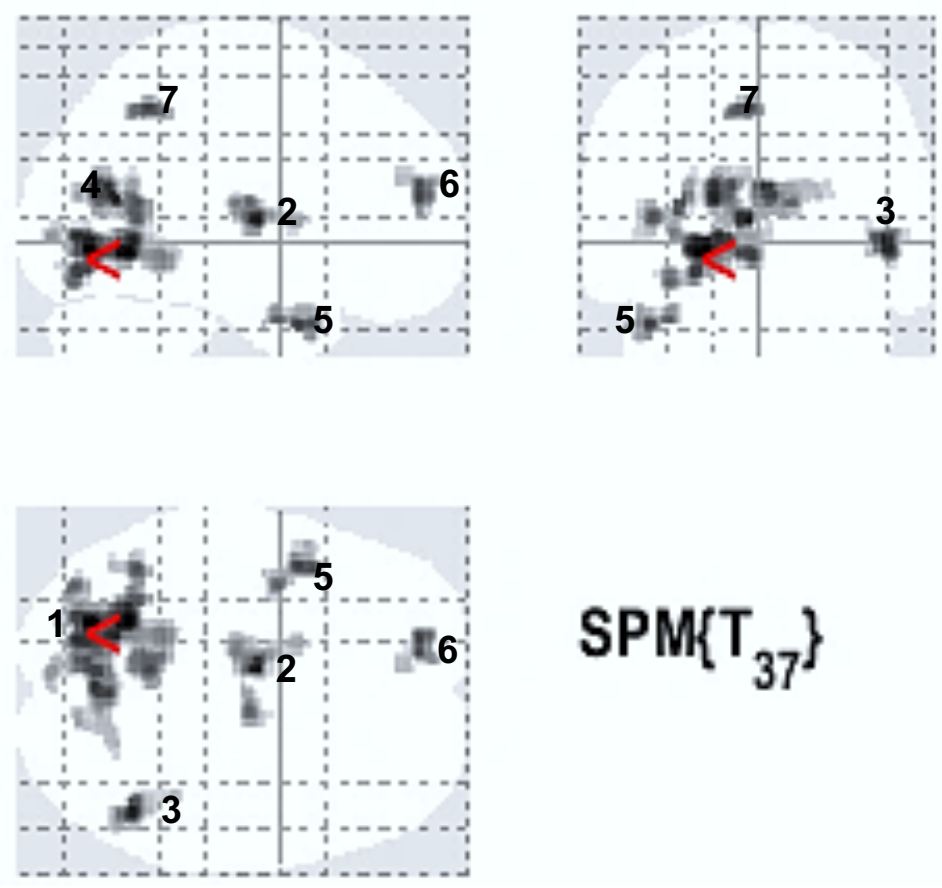

\section{$\operatorname{SPM}\left\{T_{37}\right\}$}

Figure 3. Statistical parametric maps showing brain areas that are significantly (uncorrected threshold, $p .<0.001 ; k=100$ voxels) activated for contrast Low $>$ Normal. See Table 1 for the name of the regions

Table 1. Brain area, function, number of voxels, and peak MNI coordinates $(x, y, z)$ obtained from Low $>$ Normal contrast (uncorrected threshold, $p<0.001, k=100$ voxels).

\begin{tabular}{|c|c|c|c|}
\hline Brain area & Function & Number of voxels & Peak MNI coordinates \\
\hline Left lingual gyrus (1) & $\begin{array}{l}\text { The left lingual gyrus is involved in visual retrieval, visual } \\
\text { imagery, motion imagery, and language-related semantic } \\
\text { processing [33]. It is also involved in divergence thinking that } \\
\text { influences the creative thinking process [33]. }\end{array}$ & 1215 & $-22-60-6$ \\
\hline Left thalamus (2) & $\begin{array}{l}\text { The left thalamus acts as a relay station for transmitting } \\
\text { auditory, verbal, and sensory information to prefrontal cortex } \\
\text { [34]. It is also involved in the maintenance of neural activation } \\
\text { during working memory process [34]. }\end{array}$ & 253 & $-6-106$ \\
\hline Right MTG (3) & $\begin{array}{l}\text { The right MTG processes visual stimuli and retrieves visual } \\
\text { information related to shape, color, object, scenes, face, and } \\
\text { written words [35] }\end{array}$ & 164 & $46-58-2$ \\
\hline Left calcarine (4) & $\begin{array}{l}\text { The left calcarine is associated with the visual cortex. It helps } \\
\text { to evaluate the size, shape, intensity, and color of an object in } \\
\text { the visual field [36]. }\end{array}$ & 219 & $0-7218$ \\
\hline Left MTG (5) & $\begin{array}{l}\text { The left MTG is responsible for language processing, such as } \\
\text { lexical retrieval, semantic processing, multimodal sensory } \\
\text { integration, and visual perception [37]. }\end{array}$ & 120 & $-446-32$ \\
\hline Left SFG (6) & $\begin{array}{l}\text { The left SFG is involved in high cognitive functions, such as } \\
\text { executive control and WM [38] }\end{array}$ & 133 & -165216 \\
\hline Left precuneus (7) & $\begin{array}{l}\text { The left precuneus is involved in retrieval of episodic memory, } \\
\text { self-reflection, mental imagery, and visual-spatial imagery } \\
\text { processes [39]. It also provides contextual associations } \\
\text { related to familiarity with past experiences [39]. }\end{array}$ & 129 & $-6-5248$ \\
\hline
\end{tabular}


Table 2 and 3 summarized the RsAF calculated for all the seven brain areas activated in low and normal auditory-verbal WM capacity participants, respectively. An independent samples $t$-test $(95 \% \mathrm{Cl}, p=0.05)$ showed significant RsAF differences between groups in all brain areas, except the left thalamus. The comparison results are tabulated in Table 4. The RsAF of the left lingual gyrus, right MTG, left calcarine, left MTG, left SFG, and left precuneus were significantly higher in the low auditory-verbal WM capacity group than normal auditory-verbal WM capacity group. However, left thalamus activation was not significantly different between groups.

Table 2. RsAF for low auditory-verbal WM capacity participants

\begin{tabular}{cccccccc}
\hline $\begin{array}{c}\text { Participant } \\
\text { ID }\end{array}$ & $\begin{array}{c}\text { Left lingual } \\
\text { gyrus }\end{array}$ & $\begin{array}{c}\text { Left } \\
\text { thalamus }\end{array}$ & Right MTG & $\begin{array}{c}\text { Left } \\
\text { calcarine }\end{array}$ & Left MTG & Left SFG & $\begin{array}{c}\text { Left } \\
\text { precuneus }\end{array}$ \\
\hline 003 & 0.2705 & 0.2487 & 0.2851 & 0.5211 & 0.1639 & 0.1885 & 0.3516 \\
008 & 0.2684 & 0.3968 & 0.2674 & 0.4800 & 0.4039 & 0.4343 & 0.4232 \\
009 & 0.2123 & 0.5414 & 0.3486 & 0.5470 & 0.1986 & 0.2472 & 0.5919 \\
010 & 0.3052 & 0.3360 & 0.3367 & 0.4756 & 0.3268 & 0.3358 & 0.3770 \\
011 & 0.3020 & 0.4775 & 0.3788 & 0.9006 & 0.3650 & 0.2688 & 0.5803 \\
014 & 0.2146 & 0.3199 & 0.2918 & 0.5086 & 0.2349 & 0.2632 & 0.4454 \\
015 & 0.2493 & 0.2492 & 0.1652 & 0.3236 & 0.2340 & 0.3911 & 0.3370 \\
017 & 0.2044 & 0.3442 & 0.1986 & 0.5215 & 0.1581 & 0.1289 & 0.3118 \\
018 & 0.2329 & 0.3304 & 0.2076 & 0.4826 & 0.1183 & 0.2891 & 0.3734 \\
019 & 0.2425 & 0.2979 & 0.2937 & 0.5997 & 0.1702 & 0.2386 & 0.3939 \\
020 & 0.2261 & 0.3613 & 0.2407 & 0.5871 & 0.1318 & 0.1411 & 0.3687 \\
023 & 0.3444 & 0.1911 & 0.3085 & 0.4529 & 0.1761 & 0.1687 & 0.4993 \\
024 & 0.2045 & 0.3899 & 0.1757 & 0.4350 & 0.4116 & 0.2162 & 0.2354 \\
027 & 0.2919 & 0.4449 & 0.3615 & 0.4002 & 0.5311 & 0.3350 & 0.6561 \\
030 & 0.2263 & 0.5172 & 0.1891 & 0.5517 & 0.4098 & 0.4744 & 0.4069 \\
031 & 0.2857 & 0.6398 & 0.5697 & 0.6270 & 0.5091 & 0.2306 & 0.4372 \\
033 & 0.1309 & 0.4320 & 0.1924 & 0.4489 & 0.4267 & 0.2297 & 0.3451 \\
035 & 0.1905 & 0.3140 & 0.2061 & 0.3918 & 0.6156 & 0.1452 & 0.1920 \\
040 & 0.2421 & 0.2932 & 0.2336 & 0.4673 & 0.5705 & 0.1944 & 0.2471 \\
Mean & 0.2550 & 0.3750 & 0.2764 & 0.5117 & 0.3240 & 0.2590 & 0.3986 \\
\hline
\end{tabular}

Table 3. RsAF for normal auditory-verbal WM capacity participants

\begin{tabular}{cccccccc}
\hline $\begin{array}{c}\text { Participant } \\
\text { ID }\end{array}$ & $\begin{array}{c}\text { Left lingual } \\
\text { gyrus }\end{array}$ & $\begin{array}{c}\text { Left } \\
\text { thalamus }\end{array}$ & Right MTG & $\begin{array}{c}\text { Left } \\
\text { calcarine }\end{array}$ & Left MTG & Left SFG & $\begin{array}{c}\text { Left } \\
\text { precuneus }\end{array}$ \\
\hline 001 & 0.1558 & 0.1858 & 0.1721 & 0.2267 & 0.1140 & 0.1315 & 0.1797 \\
002 & 0.2361 & 0.2985 & 0.1864 & 0.4459 & 0.1721 & 0.1719 & 0.3346 \\
004 & 0.2117 & 0.3001 & 0.1567 & 0.4041 & 0.1484 & 0.1643 & 0.2993 \\
005 & 0.1817 & 0.2088 & 0.1926 & 0.3434 & 0.1099 & 0.1356 & 0.3044 \\
006 & 0.2945 & 0.5720 & 0.2963 & 0.5441 & 0.3699 & 0.3055 & 0.3991 \\
007 & 0.1523 & 0.2000 & 0.1985 & 0.3220 & 0.1823 & 0.1582 & 0.2153 \\
012 & 0.1256 & 0.3211 & 0.1572 & 0.2082 & 0.2896 & 0.1307 & 0.2273 \\
013 & 0.1423 & 0.1850 & 0.1173 & 0.2892 & 0.1231 & 0.1097 & 0.1775 \\
016 & 0.2113 & 0.1906 & 0.2767 & 0.3956 & 0.1326 & 0.1746 & 0.3232 \\
021 & 0.2564 & 0.2800 & 0.1637 & 0.4646 & 0.2444 & 0.1977 & 0.4177 \\
022 & 0.2035 & 0.3390 & 0.2692 & 0.3737 & 0.1660 & 0.1551 & 0.3120 \\
025 & 0.1905 & 0.4263 & 0.1992 & 0.2794 & 0.2205 & 0.2058 & 0.2863 \\
026 & 0.1342 & 0.2325 & 0.1455 & 0.2287 & 0.3420 & 0.1103 & 0.3632 \\
028 & 0.2350 & 0.3618 & 0.2406 & 0.4524 & 0.2313 & 0.1993 & 0.3440 \\
029 & 0.1591 & 0.2631 & 0.2210 & 0.2128 & 0.1856 & 0.1936 & 0.2519 \\
032 & 0.1909 & 0.3311 & 0.2359 & 0.4668 & 0.2157 & 0.1702 & 0.2963 \\
034 & 0.1969 & 0.2631 & 0.2496 & 0.4720 & 0.1805 & 0.1887 & 0.2115 \\
036 & 0.2491 & 0.4266 & 0.2629 & 0.4781 & 0.2166 & 0.2577 & 0.3991 \\
037 & 0.1927 & 0.5805 & 0.2361 & 0.4074 & 0.2640 & 0.1854 & 0.3887 \\
039 & 0.1635 & 0.2800 & 0.1534 & 0.2525 & 0.1489 & 0.1343 & 0.3265 \\
Mean & 0.1942 & 0.3126 & 0.2065 & 0.3634 & 0.2029 & 0.1740 & 0.3029 \\
\hline
\end{tabular}


Table 4. Comparisons of the average RsAF between groups

\begin{tabular}{|c|c|c|c|c|c|c|}
\hline \multirow{2}{*}{ Brain areas } & \multicolumn{2}{|c|}{ Group } & \multicolumn{2}{|c|}{ Levene's test } & \multicolumn{2}{|c|}{ Independent samples t-test } \\
\hline & Low (mean \pm SD) & Normal (mean \pm SD) & $f$-value & $p$-value & $t$-value & $p$-value \\
\hline Left lingual gyrus & $0.25 \pm 0.07$ & $0.19 \pm 0.04$ & 1.44 & 0.240 & 3.10 & $0.004^{*}$ \\
\hline Left thalamus & $0.37 \pm 0.11$ & $0.31 \pm 0.11$ & 0.01 & 0.910 & 1.71 & 0.100 \\
\hline Right MTG & $0.28 \pm 0.10$ & $0.21 \pm 0.05$ & 3.79 & 0.060 & 2.84 & $0.007^{*}$ \\
\hline Left calcarine & $0.51 \pm 0.12$ & $0.36 \pm 0.10$ & 0.22 & 0.640 & 4.11 & $<0.001^{*}$ \\
\hline Left MTG & $0.32 \pm 0.16$ & $0.20 \pm 0.07$ & 19.46 & $<0.001$ & 3.06 & $0.004^{*}$ \\
\hline Left SFG & $0.26 \pm 0.10$ & $0.17 \pm 0.05$ & 7.52 & 0.009 & 3.48 & $0.001^{*}$ \\
\hline Left precuneus & $0.40 \pm 012$ & $0.30 \pm 0.10$ & 2.50 & 0.010 & 3.01 & $0.010^{*}$ \\
\hline
\end{tabular}

*Significant at $p<0.05$

\section{Discussion}

The main objective of this study was to observe any significant differences in resting-state brain activation between normal and low auditory-verbal WM capacity groups. The results of this study accept the earlier hypothesis that individuals with normal auditory-verbal WM capacity have lower resting-state brain activations than individuals with low auditory-verbal WM capacity. Low auditory-verbal WM capacity group showed significantly higher brain activations than normal auditory-verbal WM capacity group in the left lingual gyrus, bilateral MTG, left calcarine, left SFG and left precuneus. Although the low auditoryverbal WM capacity group demonstrated higher left thalamus activation than normal auditory-verbal WM capacity group, the difference was not significant. Apart from that, the brain activation results clearly exhibited left hemispheric dominance. This finding supports the previous studies suggesting that left hemisphere changes correlate with auditory-verbal WM processing [40, 41]. Increased activity of the prefrontal cortex (PFC) and left hemispheric lateralisation for auditory-verbal processing in no task condition suggest inefficient brain organisation in low auditory-verbal WM participants [19].

PFC activation contributes to auditory-verbal WM processing as it is responsible for early inhibitory modulation of auditory information to the primary auditory cortex [42]. PFC activation reflects an individual's auditory attention level [43]. The SFG is a part of PFC located at the lateral and superior (dorsolateral) portion of the brain responsible for auditory information encoding [44]. The SFG receives and integrates information [42]. For instance, left SFG processes spatial acoustic signal ('where' and 'who') when an individual is passively listening to auditory information [41]. The left SFG has been considered a task-positive neuron related to motor network [45]. In the resting-state brain, increased activity of the left SFG may not be observed as there is no goal-demanding task that requires participants to retain and manipulate auditory information accordingly. Increased activity of the left SFG has been identified as a mechanism to compensate for reduced inferior frontal gyrus (IFG) activation in low auditory-verbal WM participants with mild cognitive impairment [46]. The IFG plays an important role in the comprehension of verbal information ('what'). However, in our case, activation of left IFG in the low auditory-verbal WM participants was deemed optimal as there were no significant differences in the left IFG activation between groups.

The results also show significant differences in the activations of bilateral MTG. MTG has been associated with semantic control task-related neuron that may not show increased activation when the brain is at rest. Increase activation of the MTG was identified in the resting-state brain with a mild cognitive impairment, whereby the increased MTG activation in participants with mild cognitive impairment was linked with unilateral (right or left) MTG involvement [35]. The exact role of MTG should be studied further to understand how its activity affects the auditory-verbal WM in both healthy and clinical population.

Left lingual gyrus and left calcarine are related to visual information processing and associated with the primary visual cortex (suggested V1) needed for mental and visual imagery [33]. Visual and auditory 
regions are interconnected. Therefore, increased activations of these regions may reflect recruitment of the left SFG to process acoustic information [38]. However, a further study investigating functional connectivity between left SFG, left lingual gyrus and left calcarine is warranted. Precuneus is located at the posterior visual cortex [39]. Increase precuneus activation indicates increased activation of default mode network (DMN) related to personal memories [39] in low auditory-verbal WM capacity participants.

The main limitation of this study is that we only reported brain activations. Hence, we could not comment on the resting-state brain connectivity pathway between individuals with normal and low auditory-verbal WM capacity. This information is essential to understand better how the brain network connectivity is different in low auditory-verbal WM capacity participants than normal auditory-verbal WM capacity participants. Future work should continue this study on functional or effective connectivity.

\section{Conclusions}

This study compared brain activations of normal and low auditory-verbal WM capacity participants by means of activation maps and RsAF. Low auditory-verbal WM capacity participants showed higher activation of left lingual gyrus, bilateral MTG, left calcarine, left SFG, and left precuneus. It is suggested that the higher activations of these brain areas at rest for low auditory-verbal WM capacity group were associated with lower neural adaptability according to a required condition in low auditory-verbal WM capacity participants. For instance, higher activation of task-related neuron is not necessarily needed in the resting-state brain but is evident in low auditory-verbal WM capacity group. Hence, this study contributes to a better understanding on brain regions associated with auditory-verbal working memory components in healthy subjects. Future study on connectivity analysis can provide insight about possible differences in auditory pathway of individuals with different auditory-verbal WM capacity.

\section{Data availability}

The experimental fMRI data are owned by the Universiti Kebangsaan Malaysia (UKM). Upon approval of the respective authority in UKM, the data can be obtained from the corresponding author of this study (nazlimtrw@ukm.edu.my).

\section{Conflicts of interest}

The authors declare that there is no conflict of interest regarding the publication of this paper.

\section{Funding statement}

This research was funded by the UKM Incentive Research Grant (GGP-2017-010).

\section{Acknowledgments}

The authors would like to thank Mohamad Nor Affendi Awang and Nooradila Zolkiflee, the MRI Technologists of the Universiti Kebangsaan Malaysia Medical Centre (UKMMC), for their assistance in the rs-fMRI scanning and the Department of Radiology, UKMMC for the permission to use the MRI scanner.

\section{References}

[1] Z. Ren, Y. Zhang, H. He, Q. Feng, T. Bi, and J. Qui, "The Different Brain Mechanisms of Object and Spatial Working Memory: Voxel-Based Morphometry and Resting-State Functional Connectivity," Front. Hum. Neurosci. vol. 13, no. 248, pp. 1 - 9, 2019.

[2] A. D. Baddeley and G. Hitch, "Working Memory," Psychol. Learn. Motiv. vol. 8, pp.47-89, 1974.

[3] Z. Deldar, C. Gevers-Montoro, A. Khatibi and L. Ghazi-Saidi, "The Interaction between Language and Working 
Memory: A Systematic Review of fMRI Studies in the Past Two Decades," AIMS Neurosci. vol. 8, no. 1, pp. 1 32, 2021.

[4] C. Rogalsky, W. Matchin and G. Hickok, "Broca's Area, Sentence Comprehension, and Working Memory: An fMRI Study," Front Hum Neurosci, vol. 2, Article ID 14, 2008

[5] V. Ferpozzi, L. Fornia, M. Montagna et al., "Broca's Area as a Pre-Articulatory Phonetic Encoder: Gating the Motor Program," Front Hum Neurosci vol. 12, Article ID 64, 2018.

[6] F. Aboitiz, "Gestures, Vocalizations, and Memory in Language Origins," Front Evol Neurosci, vol. 4 Article ID 2 , 2012.

[7] P. Bublak, U. Muller, G. Gron, M. Reuter, and D. Y. Von Cramon, "Manipulation of Working Memory Information Is Impaired in Parkinson's Disease and Related to Working Memory Capacity," Neuropsychology, vol. 16, no. 4, pp. 577-590, 2002.

[8] J. H. Yoon, M. J. Minzenberg, S. Ursu et al., "Association of Dorsolateral Prefrontal Cortex Dysfunction with Disrupted Coordinated Brain Activity in Schizophrenia: Relationship with Impaired Cognition, Behavioral Disorganization, and Global Function," Am J Psychiatry, vol. 165, no. 8, pp. 1006-1014, 2008.

[9] P. Liang, Z. Wang, Y. Yang, X. Jia and K. Li, "Functional Disconnection and Compensation in Mild Cognitive Impairment: Evidence from Dlpfc Connectivity Using Resting-State fMRI," PLoS One, vol. 6, no. 7, Article ID e22153, 2011.

[10] K. R. Mcleod, L. M. Langevin, B. G. Goodyear and D. Dewey, "Functional Connectivity of Neural Motor Networks Is Disrupted in Children with Developmental Coordination Disorder and Attention-Deficit/Hyperactivity Disorder," Neuroimage Clin, vol. 4, pp. 566-575, 2014.

[11] M. A. Erickson, B. Hahn, C. J. Leonard et al., "Impaired Working Memory Capacity Is Not Caused by Failures of Selective Attention in Schizophrenia," Schizophr. Bull., vol. 41, no. 2, pp. 366-373, 2015.

[12] A. M. Kirova, R. B. Bays and S. Lagalwar, "Working Memory and Executive Function Decline across Normal Aging, Mild Cognitive Impairment, and Alzheimer's Disease," Biomed. Res. Int., vol. 2015, Article ID 748212, 2015.

[13] N. Cowan, 'The Magical Mystery Four: How Is Working Memory Capacity Limited, and Why?," Curr Dir Psychol Sci, vol. 19, no. 1, pp. 51-57, 2010

[14] P.A. Carpenter, M. A. Just and P. Shell, "What One Intelligence Test Measures: A Theoretical Account of the Processing in the Raven Progressive Matrices Test," Psychol Rev, vol. 97, no. 3, pp. 404-431, 1990.

[15] M. K. Bleckley, F. T. Durso, J. M. Crutchfield, R. W. Engle and M. M. Khanna, "Individual Differences in Working Memory Capacity Predict Visual Attention Allocation," Psycho B Rev, vol. 10, no. 4, pp. 884-889, 2003.

[16] A. I. Abd Hamid, A. N. Yusoff, S. Z. S. Mukari, amd M. Mohamad, "Brain Activation During Addition and Subtraction Tasks in-Noise and in-Quiet. Malaysian," J Med Sci, vol. 18, no. 2, pp. 3-15, 2011.

[17] M. Daneman and P. M. Merikle, "Working Memory and Language Comprehension: A Meta-Analysis," Psychon Bull Rev, vol. 3, no. 4, pp. 422-433, 1996.

[18] N. Unsworth and R. W. Engle, "Individual Differences in Working Memory Capacity and Learning: Evidence from the Serial Reation Time Task," Mem Cognition, vol. 33, no. 2, pp. 213-220, 2005.

[19] M. Daneman and P. A. Carpenter, "Individual Differences in Working Memory and Reading," J Verb Learn Verb Be, vol. 19, pp. 450-466, 1980.

[20] M. Lazar, "Working Memory: How Important Is White Matter," Neuroscientist, vol. 23, no. 2, pp. 197-210, 2017.

[21] R. Ortega, V. Lopez, X. Carrasco et al., "Neurocognitive Mechanisms Underlying Working Memory Encoding and Retrieval in Attention-Deficit/Hyperactivity Disorder" Sci Rep, vol. 10, no. 1, Article ID 7771, 2020.

[22] C. S. Prat and M. A. Just, "Exploring the Neural Dynamics Underpinning Individual Differences in Sentence Comprehension," Cereb Cortex, vol. 21, no. 8, pp. 1747-1760, 2011.

[23] E. Othman, A. N. Yusoff, M. Mohamad, H. Abdul Manan, A. I. Abd Hamid and V. Giampietro, "Effects of White Noise on Word Recall Performance and Brain Activity in Healthy Adolescents with Normal and Low Auditory Working Memory" Exp Brain Res, vol. 238, no. 4, pp. 945-956, 2020.

[24] R. Jamaluddin, Z. Othman, K. I. Musa and M. N. M. Alwi, "Validation of the Malay Version of Auditory Verbal Learning Test (MVAVLT) Among Schizophrenia Patients in Hospital Universiti Sains Malaysia (HUSM), Malaysia," ASEAN J. Psychiatr, vol. 10, no. 1, pp. 54-74, 2009.

[25] E. Othman, A. N. Yusoff, M. Mohamad et al., "Resting-state fMRI: comparing default mode network connectivity between normal and low auditory working memory groups" J Phys Conf Ser, vol. 1248, Article ID 012005, 2019.

[26] E. Othman, A. N. Yusoff, M. Mohamad and H. Abdul Manan, "The relationship between frontotemporal effective connectivity and performance during auditory working memory task in noise," J Phys Conf Ser, vol. 1497, Article ID 012011, 2020.

[27] T. Nguyen, O. Babawale, T. Kim et al., "Exploring Brain Functional Connectivity in Rest and Sleep States: A fNIRS Study," Sci Rep, vol. 8, no. 1, Article ID 16144, 2018.

[28] N. S. A. Mohd Nawi, A. A. Rahmad, K. Abdul Hamid et al., "Effective Connectivity of a Default Mode Network In Human Brain: In Search of a Dominant Node Using Spectral Dynamic Causal Modeling," Phys. Technol. Med, vol. 1, no. 1, pp. 1-14, 2020.

[29] S. Esmenio, J. M. Soares, P. Oliveira-Silva et al., "Using Resting-State DMN Effective Connectivity to Characterize the Neurofunctional Architecture of Empathy," Sci. Rep., vol. 9, no. 1, Article ID, 2603, 2019. 
[30] X. Di, and B. B. Biswal, "Identifying the Default Mode Network Structure Using Dynamic Causal Modeling on Resting-State Functional Magnetic Resonance Imaging," Neuroimage, vol. 86, pp. 53-59, 2014.

[31] A. Shmuel and D. A. Leopold, "Neuronal Correlates of Spontaneous Fluctuations in fMRI Signals in Monkey Visual Cortex: Implications for Functional Connectivity at Rest," Hum Brain Mapp, vol. 29, no. 7, pp. 751-761, 2008

[32] M. E. Raichle and M. A. Mintun, "Brain Work and Brain Imaging," Annu Rev Neurosci, vol. 29, pp. 449-76, 2006.

[33] L. Zhang, L. Qiao, Q. Chen et al., "Gray Matter Volume of the Lingual Gyrus Mediates the Relationship between Inhibition Function and Divergent Thinking," Front Psychol, vol. 7, Article ID 1532, 2016.

[34] S. E. Nadeau, "The Thalamus and Working Memory," J Int Neuropsychol Soc, vol. 14, no. 5, pp. 900-901, 2008.

[35] L. Papeo, B. Agostini and A. Lingnau, "The Large-Scale Organization of Gestures and Words in the Middle Temporal Gyrus,” J Neurosci, vol. 39, no. 30, pp. 5966-5974, 2019.

[36] P. Johns, 2014. Functional Neuroanatomy. Clinical Neuroscience, pp. 27-47, 2014

[37] T. Onitsuka, M. E. Shenton, D. F. Salisbury et al., "Middle and Inferior Temporal Gyrus Gray Matter Volume Abnormalities in Chronic Schizophrenia: An Mri Study," Am J Psychiatry, vol. 161, no. 9, pp. 1603-1611, 2004.

[38] F. Du Boisgueheneuc, R. Levy, E. Volle et al., Functions of the Left Superior Frontal Gyrus in Humans: A Lesion Study," Brain, vol. 129, no. 12, pp. 3315-3328, 2006.

[39] N. Mashal, T. Vishne and N. Laor, "The Role of the Precuneus in Metaphor Comprehension: Evidence from an fMRI Study in People with Schizophrenia and Healthy Participants," Front Hum Neurosci, vol. 8, Article ID 818, 2014.

[40] G. M. Bidelman, J. A. Brown and P. Bashivan, "Auditory Cortex Supports Verbal Working Memory Capacity," Neuroreport, vol. 32, no. 2, pp. 163-168, 2021.

[41] E. Othman, A. N. Yusoff, M. Mohamad et al., "Hemispheric Lateralization of Auditory Working Memory Regions during Stochastic Resonance: An fMRI Study," J Magn Reson Imaging, vol. 51, no. 6, pp. 1821-1828, 2019.

[42] Y. C. Chen, Y. Feng, J. J. Xu et al., "Disrupted Brain Functional Network Architecture in Chronic Tinnitus Patients," Front Aging Neurosci, vol. 8, Article ID: 174, 2016.

[43] L. Hausfeld, L. Riecke and E. Formisano, "Acoustic and Higher-Level Representations of Naturalistic Auditory Scenes in Human Auditory and Frontal Cortex," Neuroimage, vol. 173, pp. 472-483, 2018.

[44] Y. H. Chen, B. Howell, J. C. Edgar et al., "Associations and Heritability of Auditory Encoding, Gray Matter, and Attention in Schizophrenia," Schizophr Bull, vol. 45, no. 4, pp. 859-870, 2019.

[45] P. Delaveau, T. Arruda Sanchez, R. Steffen et al., "Default Mode and Task-Positive Networks Connectivity During the N-Back Task in Remitted Depressed Patients with or without Emotional Residual Symptoms," Hum Brain Mapp, vol. 38, no. 7, pp. 3491-3501, 2017.

[46] W. K. Lau, M. K. Leung, T. M. Lee and A. C. Law, "Resting-State Abnormalities in Amnestic Mild Cognitive Impairment: A Meta-Analysis," Transl Psychiatry, vol. 6, no 4, Article ID e790, 2016. 